\title{
Comportements violents chez l'enfant en Ontario
} Problématique de la suspension scolaire externe, perception des parents et alternative possible Violent behaviour in Ontario children Problems with external suspension, perceptions of parents and
possible alternatives Comportamientos violentos en la niñez en Ontario El problema de la suspension escolar externa, la percepción de los padres de familia y las alternativas posibles

\section{Maryse Paquin et Marie Drolet}

Volume 32, numéro 1, printemps 2004

La violence en milieu scolaire

URI : https://id.erudit.org/iderudit/1079123ar

DOI : https://doi.org/10.7202/1079123ar

Aller au sommaire du numéro

\section{Éditeur(s)}

Association canadienne d'éducation de langue française

\section{ISSN}

0849-1089 (imprimé)

1916-8659 (numérique)

\section{Découvrir la revue}

\section{Citer cet article}

Paquin, M. \& Drolet, M. (2004). Comportements violents chez l'enfant en Ontario : problématique de la suspension scolaire externe, perception des parents et alternative possible. Éducation et francophonie, 32(1), 201-223. https://doi.org/10.7202/1079123ar

\section{Résumé de l'article}

Depuis quelques années, nous assistons à un mouvement de durcissement des politiques et des programmes gouvernementaux visant à contrer la violence chez les jeunes. Initié dans la foulée d'événements tragiques où des adolescents ont fait feu sur d'autres élèves et/ou des enseignants, ce mouvement a conduit à la refonte de l'ensemble des lois et règlements touchant la jeunesse. La prévention et l'intervention précoces des comportements violents chez le jeune enfant apparaissent en lien avec l'idée d'éviter que de tels drames ne se répètent une fois qu'il sera parvenu à l'adolescence. Dans ce contexte, parmi les mesures disciplinaires dorénavant appliquées par l'école en Ontario, la suspension externe soulève des questions fondamentales : Est-elle appropriée à l'enfant de 3 à 9 ans? Permet-elle de contrer précocement ses comportements violents envers les pairs à l'école? Risque-t-elle, au contraire, d'aggraver sa situation-problème en entravant son processus de scolarisation et de socialisation? Si tel est le cas, quelles sont les mesures disciplinaires pouvant ne pas le priver de tels apprentissages? Afin de connaître la perception des parents sur ces questions, des entrevues ont été réalisées auprès de 60 familles dont le jeune enfant faisait face à une suspension ou à une possibilité de suspension externe en réponse à ses comportements violents envers les pairs, dans des écoles élémentaires de langue française de la région d'Ottawa. Les résultats révèlent que les parents désapprouvent majoritairement la suspension externe, lui préférant de beaucoup la suspension interne. De plus, ils estiment que l'école aurait avantage à appliquer des mesures disciplinaires non coercitives. Ces mesures commandent toutefois davantage d'ouverture et de souplesse que ne le permettent les cadres de la nouvelle Loi sur la Sécurité dans les écoles (MÉO, 2000a).

Tous droits réservés $@$ Association canadienne d’éducation de langue française, Ce document est protégé par la loi sur le droit d’auteur. L’utilisation des 2004 services d’Érudit (y compris la reproduction) est assujettie à sa politique d'utilisation que vous pouvez consulter en ligne.

https://apropos.erudit.org/fr/usagers/politique-dutilisation/ 


\title{
Comportements violents chez l'enfant en Ontario : problématique de la suspension scolaire externe, perception des parents et alternative possible ${ }^{1}$
}

\author{
Maryse Paquin, Ph.D.
}

Faculté d'éducation, Université d'Ottawa, Canada

\section{Marie Drolet, Ph.D.}

École de service social, Université d'Ottawa, Canada

\section{RÉSUMÉ}

Depuis quelques années, nous assistons à un mouvement de durcissement des politiques et des programmes gouvernementaux visant à contrer la violence chez les jeunes. Initié dans la foulée d'événements tragiques où des adolescents ont fait feu sur d'autres élèves et/ou des enseignants, ce mouvement a conduit à la refonte de l'ensemble des lois et règlements touchant la jeunesse. La prévention et l'intervention précoces des comportements violents chez le jeune enfant apparaissent en lien avec l'idée d'éviter que de tels drames ne se répètent une fois qu'il sera parvenu à l'adolescence. Dans ce contexte, parmi les mesures disciplinaires dorénavant appliquées par l'école en Ontario, la suspension externe soulève des questions fondamentales : Est-elle appropriée à l'enfant de 3 à 9 ans? Permet-elle de contrer précocement ses comportements violents envers les pairs à l'école? Risque-t-elle, au contraire, d'aggraver sa situation-problème en entravant son processus de scolarisa-

1. Cette recherche a reçu l'appui financier du Centre national de prévention du crime du ministère de la Justice du Canada, dans le cadre du Programme de partenariat en prévention du crime, de même que celui du Service de la recherche de I'Université d'Ottawa, dans le cadre du Programme de partenariat en sciences humaines et du Programme d'initiatives de recherche collaboratives interfacultaires. 
Comportements violents chez l'enfant en Ontario :

problématique de la suspension scolaire externe, perception des parents et alternative possible

tion et de socialisation? Si tel est le cas, quelles sont les mesures disciplinaires pouvant ne pas le priver de tels apprentissages? Afin de connaître la perception des parents sur ces questions, des entrevues ont été réalisées auprès de 60 familles dont le jeune enfant faisait face à une suspension ou à une possibilité de suspension externe en réponse à ses comportements violents envers les pairs, dans des écoles élémentaires de langue française de la région d'Ottawa. Les résultats révèlent que les parents désapprouvent majoritairement la suspension externe, lui préférant de beaucoup la suspension interne. De plus, ils estiment que l'école aurait avantage à appliquer des mesures disciplinaires non coercitives. Ces mesures commandent toutefois davantage d'ouverture et de souplesse que ne le permettent les cadres de la nouvelle Loi sur la Sécurité dans les écoles (MÉO, 2000a).

\section{ABSTRACT}

\section{Violent behaviour in ontario children: problems with external suspen- sion, perceptions of parents and possible alternatives}

Maryse Paquin, Ph.D. Professor, Faculty of Education, University of Ottawa

Marie Drolet, Ph.D., Professor, School of Social Services, University of Ottawa

For several years, there has been a tendency for government programs and policies to take a harder line against violence among young people. Initiated in the wake of tragic events in which adolescents shot other students and/or teachers, this movement has led to the reorganization of laws and regulations that concern youth. Early prevention and intervention in violent behaviour among young children seem to be related to the idea of preventing further tragedies when the child reaches adolescence. In this context, among the disciplinary measures now applied by the school in Ontario, external suspension raises the fundamental question: is it appropriate for children from 3 to 9 years old? Does it prevent violent behaviour against peers at school or to the contrary, does it aggravate the problem by hindering the child's educational and socialization process? If so, what disciplinary measures could be taken that would not deprive the student of these learning opportunities. To find out how parents feel about these questions, interviews were carried out with 60 families, whose children were in external suspension or facing possible external suspension for violent behaviour against their peers in French elementary schools in the Ottawa area. The results show that most parents disapprove of external suspension, the majority greatly preferring internal suspension. Moreover, they believe that the school should use non-coercive disciplinary measures. However, these measures require more openness and flexibility than the framework of the new school security law allows. (OME, 2000a). 
Comportements violents chez l'enfant en Ontario :

problématique de la suspension scolaire externe, perception des parents et alternative possible

RESUMEN

Comportamientos violentos en la niñez en ontario: El problema de la suspension escolar externa, la percepción de los padres de familia y las alternativas posibles

Maryse Paquin, Ph.D. Profesora, Facultad de educación, Universidad de Ottawa Marie Drolet, Ph.D.,Profesora, Facultad de educación, Universidad de Ottawa

Desde hace varios años asistimos a un movimiento de endurecimiento de las políticas y los programas gubernamentales destinados a combatir la violencia entre los jóvenes. Este movimiento se inició gracias la influencia de eventos trágicos en los cuales adolescentes acribillaron a alumnos y maestros, eventos que provocaron la reformulación del cuerpo de leyes y reglamentos sobre los jóvenes. La prevención y la intervención precoces de los comportamientos violentos entre los niños se ligan con la idea de evitar que dichos dramas no se repitan cuando los niños lleguen a la adolescencia. En dicho contexto, entre las medidas aplicadas en las escuelas en Ontario, la suspensión externa provoca el surgimiento de cuestiones fundamentales: ¿ es una medida apropiada para niños de 3 a 9 anos? ¿ Esta medida previene efectivamente los comportamientos violentos hacia los pares en la escuela? ¿Acaso no se corre el riesgo de agravar la situación problemática al obstruir el proceso de escolarización y de socialización? Si tal es el caso ¿̨cuales son las medidas disciplinarias que no ponen traba a dichos procesos? Con el fin de conocer la percepción de los padres de familia sobre estas cuestiones, se entrevistaron a 60 familias que contaban con un niño sujeto a una suspensión o con la posibilidad de ser suspendido y enviado a la casa en tanto que medida disciplinaria por sus comportamientos violentos hacia sus pares en las escuelas primarias de lengua francesa de la región de Ottawa. Los resultados demuestran que, en su mayoría, los padres de familia desaprueban la suspensión externa y prefieren la suspensión interna. Además, consideran que la escuela debería aplicar medidas no coercitivas. Este tipo de medida exige más apertura y flexibilidad que lo que permite el cuadro de la nueva ley sobre la seguridad en las escuelas (MEO, 2000a). 
Comportements violents chez l'enfant en Ontario :

problématique de la suspension scolaire externe, perception des parents et alternative possible

\section{Introduction}

La série d'événements tragiques survenus au cours des dernières années, notamment à Columbine aux États-Unis et à Erfurt en Allemagne, où des adolescents ont eu recours à des armes à feu pour régler leurs comptes avec des pairs et/ou des enseignants, a forcé les autorités du monde entier à réfléchir sur la manière de mieux les contrer (Lemonick, 2002). Toutefois, les échecs au niveau des efforts consentis pour éviter la répétition de tels drames, ont conduit naturellement à l'idée de la prévention et de l'intervention précoces des comportements violents. Selon les résultats de certaines recherches longitudinales, il semble que les comportements de l'enfant représentent de précieux indicateurs quant à la manière probable dont il agira à l'adolescence (Nagin et al., 2001; Tremblay, 1995; Rich, 1992), et même à l'âge adulte (Kratzer et Hodgins, 1997).

\section{Contexte canadien et ontarien}

Le Canada n'a pas échappé à cette prise de conscience puisque, selon Viau (2001), au cours de la dernière décennie, il affichait le taux de mises sous garde d'adolescents délinquants le plus élevé de tous les pays occidentaux, y compris des ÉtatsUnis. Dans le but de faire une distinction entre le grand nombre d'infractions mineures commises par des adolescents et le petit nombre d'infractions graves avec violence, le gouvernement canadien procédait, en 1998, à la refonte de la Loi sur les jeunes contrevenants (MJC, 1998). Puis, en 2002, il procédait à l'adoption de la nouvelle Loi sur le système de justice pénale pour les adolescents (MJC, 2002). Cette nouvelle loi fédérale donne au tribunal pour adolescents le pouvoir d'imposer des peines d'adultes de plus de deux ans d'emprisonnement dès l'âge de 14 ans, soit deux ans de moins que l'âge qui était requis auparavant.

De son côté, dans la foulée du mouvement de durcissement des politiques et des programmes fédéraux visant la prévention et l'intervention précoces des comportements violents, l'Ontario procédait à l'adoption de la nouvelle Loi sur la Sécurité dans les écoles (MÉO, 2000a). Cette nouvelle loi autorise l'application de mesures coercitives et dissuasives dès l'apparition, chez l'enfant, des premiers comportements jugés inappropriés en milieu scolaire. Parmi ces mesures, la suspension scolaire externe peut dorénavant être appliquée dès l'âge de 3 ans. Avant l'entrée en vigueur de cette nouvelle loi, intervenir face à la violence dans les écoles ontariennes signifiait déjà le fait de se conformer à la Politique de Tolérance Zéro, adoptée en 1994, par le ministère de l'Éducation et de la Formation (MÉFO, 1994). De cette politique découlait l'obligation, pour le milieu scolaire, de se doter d'un code de conduite prévoyant les diverses conséquences face aux comportements violents des élèves. L'une de ces conséquences était l'imposition de la suspension externe dès la première infraction jugée grave. Les normes de gravité établies par le Code de conduite des écoles correspondent généralement à celles du Conflits Tactics Scale (Strauss et 
Comportements violents chez l'enfant en Ontario:

problématique de la suspension scolaire externe, perception des parents et alternative possible

Gelles, 1990). Cette échelle américaine détermine deux catégories et sous-catégories de comportements violents, soit les verbaux et les physiques, mineurs et graves. À titre d'exemple, le comportement violent physique grave le plus fréquent, justifiant la suspension externe chez les enfants de 3 à 9 ans aux États-Unis, est celui de frapper un pair avec force et blessures, soit de donner un coup de pied, un coup de poing ou de mordre (Dupper et Bosch, 1996). Cette situation est très comparable à celle qui prévaut actuellement dans les écoles canadiennes (Vitaro et Gagnon, 2000).

Pour de tels comportements violents, l'Ontario autorise la suspension externe jusqu'à concurrence de vingt jours ouvrables au cours d'une même année scolaire (MÉFO, 1994). Au delà de ce nombre, le renvoi de l'école peut être envisagé pour une période d'une année si aucun changement n'est observé suite à des interventions répétées auprès de l'enfant et de sa famille (MÉO, 2000a). Le renvoi débute généralement vers l'âge de 9 ans et implique un changement d'école. Actuellement, les élèves peuvent faire face à deux types de renvoi : partiel ou complet. Les élèves faisant face à un renvoi partiel de leur école sont généralement dirigés vers des écoles et des classes spéciales, selon les termes de la Section 20 de la Loi sur l'éducation de l'enfance en difficulté (MÉO, 2001b). Quant aux élèves faisant face à un renvoi complet de toute école de l'Ontario financée par des fonds publics, ils ont l'obligation depuis peu de s'acquitter avec succès d'un programme de discipline rigide afin d'y être admis à nouveau. Pour répondre à ce nouveau besoin, une quinzaine d'écoles axées sur un tel programme disciplinaire, que l'on surnommaient anciennement «écoles de réforme», ont été implantées à titre expérimental dans toutes les régions de l'Ontario (MÉO, 2001a).

Parallèlement à cette situation, dans le but de mieux encadrer le code des écoles, le ministère de l'Éducation de l'Ontario adoptait, en 2000, le Code de conduite provincial (MÉO, 2000b). Ce Code, en plus d'élaborer des normes claires pour assurer la sécurité de toute personne se trouvant dans une école, rend dorénavant obligatoire et immédiate la suspension externe, non seulement dans des cas de violence physique mais également dans le cas de violence verbale, consistant notamment à menacer de causer des dommages corporels graves. À cette mesure s'ajoute le nouveau pouvoir des directions d'écoles et du personnel enseignant de suspendre ou de renvoyer les élèves dans le cas d'infractions graves, notamment en raison d'une agression physique causant des dommages corporels à autrui et nécessitant les soins d'un professionnel de la santé.

Bref, c'est dans le cadre de ces nouvelles lois et règlements, tant fédéraux que provinciaux, que les écoles ontariennes doivent dorénavant envisager la gestion des comportements violents. C'est également dans ce contexte socio-politique que le milieu scolaire risque fort, au cours des prochaines années, de connaître un phénomène de recrudescence de la suspension externe, en réponse aux comportements violents des enfants envers leurs pairs à l'école. Si l'entrée en vigueur de toutes ces nouvelles lois et règlements a permis de mieux encadrer les conséquences face aux actes posés par ces derniers, une difficulté persiste toujours, tant dans le milieu scolaire que dans la société en général, soit celle de s'entendre sur une définition commune de ce qu'est un comportement violent. 
Comportements violents chez l'enfant en Ontario:

problématique de la suspension scolaire externe, perception des parents et alternative possible

\section{Comportements violents : de multiples définitions}

Les comportements violents peuvent être définis de multiples façons selon les perspectives adoptées par les chercheurs. Selon Duhamel-Maples (1996: 41) : «Une multitude d'appellations sont généralement utilisées pour désigner le comportement violent chez l'enfant, et ce, dans au moins six champs professionnels ». Même si ces appellations sont parfois utilisées comme s'il s'agissait de concepts interchangeables, elles font toutefois références à des réalités fort différentes, telles que décrites dans les ouvrages consultés.

En pédopsychiatrie, selon le DSM-IV (APA, 1996 : 63), il est notamment question du Trouble du déficit de l'attention avec hyperactivité (TDAH) et impulsivité (TDAHI). Les trois composantes de ce trouble réfèrent à la difficulté chez l'enfant de se concentrer, jumelée à un haut niveau d'activité et à un manque de contrôle de soi. Dans le DSM-IV (APA, 1996: 68), il est également question du Trouble oppositionnel avec provocation (TOP), qui comprend un ensemble de comportements négativistes, hostiles et provocateurs: colère, contestation, opposition, susceptibilité, attitude blâmante, méchanceté, etc. Le DSM-IV (APA, 1996 : 66) identifie aussi le Trouble des conduites (TC), qui semble même être de loin le plus grave problème pouvant affecter la vie de l'enfant (Gagnon et Vitaro, 2000). Ce trouble réfère à un ensemble de conduites, répétitives et persistantes, dans lequel sont bafoués les droits fondamentaux d'autrui ou les normes et règles sociales, telles que la cruauté physique envers les personnes ou les animaux, la destruction de biens matériels, la violation graves de règles établies, etc. Finalement, un quatrième trouble recensé (APA, 1996 : 69) concerne le Comportement perturbateur (TCP). Ce dernier se situe à mi-chemin entre le Trouble oppositionnel avec provocation et le Trouble des conduites. Basé sur une approche clinique, l'ensemble de ces troubles «sont définis à partir de symptômes ayant persisté pendant au moins six mois, à un degré qui est inadapté et ne correspond pas au niveau de développement de l'enfant» (APA, 1996: 63).

En psychologie, plusieurs termes ont été répertoriés, soit les désordres, les handicaps ou les perturbations d'ordre émotionnel (Kauffman, 1997). Ceux-ci réfèrent à des conditions de souffrance morale chez l'enfant qui sont soit ponctuelles, soit persistantes. Elles comprennent l'anxiété, la maniaco-dépression, l'excessivité/compulsivité, etc. Toutefois, c'est le terme agressivité qui semble le plus couramment utilisé pour décrire l'état psychologique de l'enfant (Christenson et al., 1997). Les plus récentes taxonomies divisent même les comportements agressifs selon leur caractère internalisé ou externalisé (Gagnon et Vitaro, 2000). Notamment, l'agressivité externalisée se subdivise en deux types, soit les comportements réactifs (sans provocation) et proactifs (avec provocation).

En ce qui a trait au domaine du service social, la violence chez les enfants est abordée davantage sous l'angle de l'antisociabilité (Patterson, 1992) et des comportements inadaptés socialement (Bishop et Rothbaum, 1992). Ces comportements réfèrent à la difficulté d'établir des relations sociales satisfaisantes, de manière individuelle ou collective. L'absence d'habiletés ou de compétences sociales chez l'enfant pouvant mener au harcèlement et au rejet par les pairs. 
Comportements violents chez l'enfant en Ontario :

problématique de la suspension scolaire externe, perception des parents et alternative possible

Dans le domaine de l'éducation, il est plutôt question de problèmes, de difficultés ou de troubles de comportement (Fortin et al., 1996). Ceux-ci réfèrent à l'incapacité chez l'enfant de fonctionner de manière «normale» dans le cadre des règles fixées par l'institution scolaire. Il est également question d'indiscipline (MÉQ, 1992), un terme pour lequel le personnel enseignant est amené à exercer la gestion de classe. Selon Jeffrey et Simard (2000), celle-ci est définie comme étant un travail qui touche notamment aux règles de la classe et aux conséquences à leur manquement, de même qu'aux routines de fonctionnement et à leur ordonnancement. Selon ces mêmes auteurs, les premières semaines de l'année scolaire se révèlent particulièrement critiques puisque c'est au cours de cette période que les enseignants tentent d'anticiper et de prévenir les problèmes liés à cette gestion. À cet effet, ils consacrent beaucoup de temps à communiquer les règles, les procédures et leurs attentes face aux comportements des élèves. C'est également au cours de cette période qu'ils cherchent à se faire une idée sur chacun d'eux.

Quant au domaine de la psychopédagogie, les plus récentes études retiennent le concept d'enfants à risque (Paquin et Drolet, 2000). Ce concept s'intéresse plus particulièrement à la possibilité qu'un enfant devienne tour à tour un agresseur ou une victime de la violence. À ce concept, sont associés ceux des facteurs de protection et de résilience. Selon Fortin et al. (1996), qui étudie la question sous des angles multifactoriels, les comportements violents sont le résultat de l'accumulation d'un certain nombre de facteurs de risque qui augmentent la probabilité de voir apparaître la situation-problème chez l'enfant. Par ailleurs, la présence d'un certain nombre de facteurs de protection diminuent la probabilité de la voir émerger. Quant aux facteurs de résilience, ils correspondent à la capacité de l'enfant de faire face à des conditions adverses avec succès.

Finalement, dans le domaine de la criminologie, en contrevenant aux règles, à l'ordre et aux lois en vigueur, l'enfant violent est considéré comme étant un futur délinquant (Leblanc, 1999), ainsi qu'un criminel de demain (Carrington, 1999).

Tous ces termes illustrent bien la diversité conceptuelle qui règne autant entre les différents domaines qu'au sein d'un même champ professionnel. D'où l'importance de préciser la définition de comportement violent qui a été privilégiée dans la présente recherche.

Selon Strauss et Gelles (1990), concepteurs du Conflits Tactics Scale, le comportement violent signifie : «Toute action menée avec l'intention réelle ou perçue de blesser une autre personne physiquement, matériellement ou symboliquement». Comme la notion d'intention occupe une place prépondérante dans cette définition et qu'elle ne tient pas assez compte du résultat de cette intention, Bouchard et Tessier (1996) proposent la variante suivante, soit : « Toute action qui, dans une situation de conflit, compromet ou risque de compromettre l'intégrité ou le bien-être psychologique et/ou physique de la personne». Les chercheures ont privilégié la seconde définition parce qu'elle rejoint davantage l'idée que l'enfant n'est pas toujours conscient des conséquences aux gestes posés, qu'ils soient prémédités ou non. 
Comportements violents chez l'enfant en Ontario :

problématique de la suspension scolaire externe, perception des parents et alternative possible

\section{Conflits : origines multiples mais conséquence unique}

Si l'on s'entend pour dire que dans le milieu scolaire, les conflits entre les élèves semblent inévitables et que leurs origines tout autant que leurs causes soient multiples, les comportements violents qui en découlent entraînent généralement une conséquence unique: la suspension externe. Largement utilisée malgré le manque de connaissances sur ses effets en bas âge, cette mesure disciplinaire est non seulement appliquée en réponse aux comportements violents mais également pour des motifs de non respect du code de conduite. Selon Van Neste (1994), cette situation peut expliquer à elle seule le nombre de plus en plus élevé d'élèves suspendus à répétition, et ce, parfois pour des raisons relativement banales. Tant et si bien que son utilisation fait régulièrement l'objet de remises en question.

\section{Suspension scolaire externe : les "pour " et les " contre "}

Les défenseurs de l'utilisation de la suspension externe fondent leur argumentation sur le fait que l'ordre et les droits de la majorité des élèves se doivent d'être protégés. À ce titre, il est généralement reconnu que les élèves ayant des comportements violents envers leurs pairs à l'école influencent négativement les relations interpersonnelles (Lauwrence et Hayden, 1995). De plus, ils nuisent au climat d'apprentissage dans la classe et contribuent significativement à l'augmentation du niveau de stress chez les enseignants (Imich, 1994). Ce qui prive la majorité des élèves de recevoir un enseignement de qualité. Lors de situations de crise, la suspension permet alors à l'enseignant et aux élèves de la classe, de même qu'à l'élève suspendu, d'apaiser temporairement les tensions (Garibaldi, 1996).

Quand à ses opposants, ils maintiennent que lorsqu'il y a protection des besoins éducationnels et des droits de la majorité des élèves, il y a nécessité d'étendre cette protection à la minorité d'élèves aux prises avec une problématique de comportements violents (Mellard et Seybert, 1996). C'est pourquoi, le comportement violent doit être entendu non seulement comme étant une condition perturbatrice pour l'ensemble des élèves mais également comme étant une limite au développement scolaire et social de l'élève suspendu. Berkowitz (1993) définit cette limite comme étant "une absence de contrôle interne permettant à l'élève de s'ajuster face aux demandes formulée par l'école et l'absence de compétences lui permettant d'y répondre adéquatement ». Quoi qu'il en soit, dans la grande majorité des cas, la suspension externe est le reflet d'une discipline déficiente et de tensions élevées dans l'univers scolaire (Uchitelle et al., 1989).

Même si un lien peut être établi entre son application et les nouvelles approches de protection et de sécurité préconisées tant par le ministère fédéral de la Justice que le ministère provincial de l'Éducation, cette mesure coercitive apparaît rapidement comme une solution temporaire à un problème beaucoup plus complexe, soit celui de ne pas compromettre le développement scolaire et social des enfants (CCDS, 2001), à plus forte raison lorsqu'ils sont aux prises avec une problématique de comportements violents envers leurs pairs à l'école. 
Comportements violents chez l'enfant en Ontario :

problématique de la suspension scolaire externe, perception des parents et alternative possible

Bien que la suspension scolaire externe soit une mesure dont l'utilisation est dorénavant encadrée par des lois, règlements et politiques tant ontariennes que canadiennes, il y a tout lieu de se demander si, dans sa forme actuelle, c'est-à-dire appliquée de manière uniforme et sans distinction pour l'ensemble des comportements violents des élèves, elle s'avère efficace. Il y a également lieu de s'interroger si elle permet aux élèves de 3 à 9 ans de surmonter leur situation-problème, tout en leur permettant de faire l'apprentissage de compétences scolaires et sociales nécessaires au développement de relations plus harmonieuses avec leurs pairs, voire de s'adapter aux multiples exigences de l'école. D'où les questions de recherche formulées par les chercheures.

\section{Questions de recherche}

Parmi l'ensemble des mesures disciplinaires dorénavant appliquées par le système scolaire ontarien, afin de contrer les comportements violents des enfants envers leurs pairs à l'école, la suspension scolaire externe peut-elle contribuer de manière précoce à lui faire surmonter sa situation-problème? Risque-t-elle, au contraire, de l'aggraver en entravant son processus de scolarisation et de socialisation? Si tel est le cas, quelles sont les mesures disciplinaires permettant de ne pas priver les élèves de tels apprentissages?

Afin de connaître la perception des parents sur ces questions, des entrevues ont été réalisées auprès de 60 familles francophones dont le jeune enfant faisait face à une suspension ou à une possibilité de suspension externe en réponse à ses comportements violents envers les pairs, dans des écoles élémentaires de langue française de la région d'Ottawa. La plupart des familles ont participé sur une base volontaire à partir d'une invitation lancée par leur conseil scolaire et/ou leur direction d'école. D'autres ont été référées par un organisme d'intervention sociale oeuvrant auprès des familles francophones, le Centre psychosocial pour enfants et familles. Enfin, quelques unes ont participé suite à une invitation publique parue dans les journeaux.

\section{Méthode de collecte et d'analyse de données}

De juin 2000 à juin 2002, une trentaine d'entrevues structurées (à l'aide d'une grille comprenant des questions ouverte et fermées) et une trentaine d'entrevues semistructurées (en profondeur) ont été réalisées. Les entrevues ont été enregistrées puis ont été transcrites sur traitement de texte. Finalement, leur contenu a été codifié à l'aide du logiciel N-VIVO, version 1.3, puis soumis à l'analyse qualitative (L'Écuyer, 1987). 
Comportements violents chez l'enfant en Ontario:

problématique de la suspension scolaire externe, perception des parents et alternative possible

\section{Données sociodémographiques}

L'échantillon d'enfants dont les parents ont participé à la recherche se compose de 6 filles et de 54 garçons. Cette répartition entre les sexes, soit $10 \%$ de filles et $90 \%$ de garçons correspond à la situation généralement décrite dans la littérature, à savoir que les comportements violents correspondent à une problématique davantage présente chez le sexe masculin (Kauffman, 1997).

Parmi les filles, deux sont âgées entre 3 et 6 ans et quatre sont âgées entre 7 et 9 ans. Pour ce qui est des garçons, 23 sont âgés de 3 à 6 ans et 31 sont âgés de 7 à 9 ans.

Des 60 enfants, 16 sont au cycle préscolaire, soit trois à la garderie, cinq à la maternelle et neuf au jardin. Par ailleurs, 44 enfants sont au cycle primaire, soit 11 en première année, 13 en deuxième année et 19 en troisième année. L'augmentation du nombre de sujets en fonction des cycles semble également cohérente avec le fait que plus l'enfant vieillit, plus les comportements violents se cristalisent (Loeber et Farrington, 2000). La répartition entre les cycles semble également cohérente avec le fait que les comportements violents se produisent davantage lorsqu'entre en jeu le processus de scolarisation, inhérent au passage entre le cycle préscolaire et le cycle primaire (Fortin et al., 1996).

Au niveau de leur histoire scolaire, la moitié des enfants de l'échantillon éprouvent des difficultés d'apprentissage dans les matières scolaires (lecture, écriture et mathématiques), nécessitant un Programme d'enseignement individualisé (PEI) et le suivi d'une enseignante-ressource, neuf ont changé d'école afin de bénéficier de services de soutien dans une école et une classe spéciales, aussi appelée "Section 20 ", selon les termes de la Loi sur l'éducation de l'enfance en difficulté (MÉO, 2001b), et six ont doublé leur première ou leur deuxième année.

Quant à leur histoire médicale, 12 ont un diagnostic médical du Trouble déficitaire de l'attention avec hyperactivité (TDAH) nécessitant une prescription de Ritalin ${ }^{\circledR}$, 19 éprouvent divers problèmes de santé physique et/ou mentale nécessitant une médication prescrite par un professionnel de la santé. Finalement, 42 bénéficient des services d'un travailleur social ou d'un psychologue, sept reçoivent un suivi de la part d'un pédopsychiatre et cinq participent à des programmes de rééducation du langage (orthothérapie) et/ou de rééducation physique (ergothérapie).

Parmi les répondants, 55 sont des mères, dont la moyenne d'âge se situe entre 30 et 34 ans. De ce nombre, 43 occupent un emploi à temps plein sur le marché du travail ou comme travailleuse autonome, trois sont aux études à temps plein, une occupe un emploi à temps partiel, une est chômeuse et sept demeurent à la maison. Quant aux pères, au nombre de cinq, leur moyenne d'âge se situe entre 35 et 39 ans. Parmi eux, trois occupent un emploi à temps plein, un est chômeur et le statut professionnel du dernier répondant n'est pas disponible. L'échantillon comprend également une grand-mère adoptive âgée entre 50 et 54 ans qui demeure à la maison.

2. Ces seuils sont déterminés par le nombre de membres dans la famille en fonction du revenu annuel, par le Conseil national du bien-être social (2002). 
Comportements violents chez l'enfant en Ontario :

problématique de la suspension scolaire externe, perception des parents et alternative possible

$\mathrm{Au}$ niveau du statut familial, l'échantillon comprend 29 familles monoparentales, 26 familles biparentales et cinq familles reconstituées. Parmi ces familles, 15 vivent sous le seuil de la pauvreté ${ }^{2}, 17$ familles se situent dans la classe moyenne, 13 dans la classe moyenne-supérieure et 14 dans la classe supérieure. De manière cohérente avec les résultats des plus récentes études sur la pauvreté au Canada (CNBS, 2002), 12 des 15 familles de l'échantillon qui déclarent vivre sous le seuil de la pauvreté sont également monoparentales. Par ailleurs, six des 13 familles se situant dans la classe moyenne-supérieure sont également dirigées par une mère seule.

Parmi l'ensemble des mères, 15 ont fait des études de niveau universitaire, 17 de niveau collégial, 21 de niveau secondaire et deux de niveau élémentaire. Quant aux pères, un est de niveau universitaire, un de niveau collégial, deux de niveau secondaire et la scolarité du dernier répondant n'est pas disponible.

Les familles de l'échantillon sont en grande majorité canadienne-française. À ce sujet, comme langue d'usage, 29 d'entre elles déclarent parler le français à la maison, 24 parlent le français et l'anglais, tandis que sept parlent le français ainsi qu'une autre langue, soit le créole, l'espagnol, le grec, le chinois, l'arabe ainsi que deux langues africaines.

\section{Quelques résultats de recherche}

En dépit de l'entrée en vigueur des nouvelles mesures légales encadrant la suspension scolaire externe en Ontario, 39 des 60 parents de l'échantillon se déclarent en désaccord avec son application en réponse aux comportements violents de leur enfant envers leurs pairs à l'école. Parmi les 21 autres parents, 11 se disent plus ou moins en accord, six se montrent en accord et quatre déclarent ne pas avoir d'opinions précises sur le sujet. Aucun enfant de ces derniers n’a toutefois expérimenté la mesure disciplinaire.

\section{Les parents en désaccord}

Parmi les parents qui se disent en désaccord avec l'application de la suspension externe, une première raison invoquée concerne le fait que leur enfant est trop jeune pour en saisir toute la portée: "À son âge, il n'a vraiment pas compris (04); «Mon enfant ne comprend pas les conséquences de ses actions. Une journée sans aller à l'école n'est pas assez proche, assez rapide suite à un mauvais comportement. Il y a trop d'attente entre l'action et la punition (22)»; "Mon enfant ne sait même pas ce que ça veut dire être suspendu (92)»; "Ça lui passe par-dessus la tête, parce qu'il ne s'en rappelle même pas (110)».

Près de la moitié d'entre eux expliquent qu'au lieu d'avoir un effet punitif, cette mesure apparaît même plaisante aux yeux de leur enfant: "C'est une journée de vacances avec sa maman (01)"; "Je suis contre parce que mon enfant est heureux de cette situation (13)»; "Il pense que c'est une journée de congé (50)». "C'est amusant parce qu'il n'est pas obligé de faire ses travaux scolaires (58) »; Pour lui, rester à la maison, c'est une game. Et il aime ça jouer (81)»; « Ça lui fait plaisir de pas y aller (91)». 
Comportements violents chez l'enfant en Ontario:

problématique de la suspension scolaire externe, perception des parents et alternative possible

Selon certains parents, cette situation peut même encourager la récidive des comportements violents chez leur enfant : "Elle n'a qu'à péter une crise pour pouvoir venir à la maison (09)»; "Ça risque d'avoir l'effet contraire et d'encourager les mauvais comportements (11)»; "Il sait quoi faire pour avoir une suspension (51)»; "Je pense que ça va amplifier ses comportements plus que d'autre chose. [...] En lui indiquant la porte de sortie, il va l'utiliser plus souvent. Ça va devenir une façon d'aller à la maison (90) »; «Il sait que s'il fait un mauvais coup, je viendrai le chercher (97)».

Pour contrer le risque de récidive inhérent à l'effet positif que procure la suspension externe à leur enfant, certains parents proposent que l'école utilise des mesures disciplinaires à l'interne qui soient contraignantes: "Il me semble qu'il aurait pu avoir d'autres choses comme des excuses, s'arranger pour qu'il ait un peu honte (12)»; "Soit relire le code de conduite de l'école, faire de la lecture supplémentaire et faire un peu de nettoyage (96) »; "Une suspension pour moi, ce serait... des travaux forcés. Ça l'air ridicule, mais ils pourraient ramasser, faire du ménage à l'école (97) »; «Lui enlever une activité spéciale, ça ça peut faire mal (106)».

D'autres proposent que l'école applique des mesures davantage positives: "Je leur ai demandé de lui donner une tâche qui l'aiderait à se sentir revalorisé. Il adorerait laver les tableaux (21)"; "Je leur ai proposé de montrer quelques notes de piano à mon fils. Il adore la musique. Je leur ai dit que ça pouvait être une forme de motivation. L'école a un piano dans le gymnase qui ne sert jamais. Il n'ont jamais mis cette solution en pratique (48)».

Afin d'être efficace, des parents soutiennent également qu'il serait important que l'école utilise un système de mesures disciplinaires graduelles avant d'imposer la suspension externe: "Le directeur aurait pu utiliser d'autres techniques avant d'en arriver là. Ça aurait été plus d'ouvrage pour lui. C'est facile de renvoyer (12)»; «Sans avertissement, c'est vraiment une mesure exagérée (52)».

Afin de garder une certaine crédibilité au yeux de leur enfant, selon les parents, il faudrait que l'école cesse d'utiliser la suspension externe pour des motifs banals : «Des retenues, ça c'est normal, mais pas te faire suspendre de l'école pour des petites choses (102)»; "Je veux dire pas si c'est juste l'attitude ou juste verbal, non je trouve qu'il y a d'autres façons que celle de suspendre (106) »; "Suspendre juste parce qu'elle n'écoute pas toujours, là c'est pas correct (112)».

En ce sens, les parents jugent que l'application de la suspension externe à répétition s'avère dès plus inefficace: "C'est tout le temps les mêmes qui sont suspendus, ceux qui ont des problèmes. À quoi ça rime de faire ça. Ça donne aucun résultat (10.1)»; "L'école suspend trop souvent mon enfant. Des semaines de temps que ça dure et ça n'améliore pas la situation (51)».

Même si elle n'était utilisée qu'en dernier ressort, selon plusieurs parents, la suspension externe s'avère toujours très dommageable au plan de l'apprentissage : "L'enfant est exclu des apprentissages (10)»; "La suspension accélère les problèmes d'estime de soi de l'enfant et ses difficultés d'apprentissage dans les matières scolaires (48) »; "Les élèves prennent de plus en plus de retard et l'enseignante s'en lave les mains (52) »; "Si les enfants sont déjà en arrière à cause de leur comportement, ça les met encore plus en arrière (85)». 
Comportements violents chez l'enfant en Ontario:

problématique de la suspension scolaire externe, perception des parents et alternative possible

Les parents soutiennent qu'elle s'avère également très dommageable au plan de la socialisation de l'enfant: "L'isolement de mon enfant amène un manque au niveau de son apprentissage social (11)»; «Le fait de suspendre l'enfant, ça représente la $1^{\text {re étape }}$ pour qu'il se sente exclu et qu'il n'aille pas dans le droit chemin plus tard (48)»; «La suspension a été néfaste parce que la première chose, il a été retiré de ses amis. Sans explications (109)».

Au niveau des attitudes de leur enfant, les parents ont observé que cette mesure contribue à les démotiver tout en favorisant leur désintérêt pour l'école : «Mon enfant ne voulait pas retourner à l'école après la suspension (09)»; «C'est absolument zéro sur l'enfant car il ne veut plus retourner à l'école. De mon côté, je dois travailler deux fois plus fort pour l'encourager à y retourner. Ce n'est pas envisageable qu'un enfant soit "drop out" en première année (48)".

Dans certains cas, la manière dont la suspension externe est présentée aux parents peut même contribuer à leur faire ressentir un grand inconfort, de même que causer un réel tort moral à leur enfant: "Dans le contexte dans lequel ça été présenté, c'était un brin intimidant parce que, je peux comprendre que même si l'éducation de l'enfant c'est notre responsabilité, je me suis sentie comme pogné au collet (06) »; "Le directeur a dit que mon enfant ne pourrait plus venir à l'école [...] à sa première journée d'école. C'était un gros coup à prendre pour mon enfant. Il était très traumatisé. Il s'est mis en dessous d'un bureau et a commencer à pleurer (44)»; "La suspension a été très dure pour mon enfant... (62)»; "Un enfant de 3 ans. Veux-tu bien me dire ce qu'il a fait de si mal à 3 ans [...] pour le rejeter comme ça (109) »; "À quatre ans, c'est plus parce qu'ils veulent se débarasser d'elle (112)».

Les parents soulignent aussi comme inconvénient que la suspension externe leur occasionne davantage de désagréments à eux qu'à leur enfant car cette mesure disciplinaire les oblige à s'absenter du travail et les force à modifier leur horaire déjà chargé : "C'est moi qui manque une journée de travail (01)»; "La suspension ne punit pas l'enfant, mais plutôt les parents qui ne peuvent pas aller travailler (13)»; «Des fois, c'est un problème de savoir qui de nous deux va prendre la journée «off». Des fois, on est mal pris! (96) »; "Ça n'aide pas les parents, ça c'est sûr et certain quand tu travailles (97) ॥; «C'est un problème parce que c'est encore un changement de routine (111)».

Les parents considèrent que la suspension externe représente un transfert de responsabilité du milieu scolaire vers le milieu familial: "Le problème se passe à l'école et il devrait rester à l'école (12)"; "Je trouve que l'enseignante ne fait pas sa job. On devrait lui couper son salaire lorsqu'elle met l'enfant en dehors de la classe. Elle serait bien plus motivée pour le garder (48)».

D'une certaine manière, ce transfert exige aux parents de transférer eux aussi leurs responsabilités parentales à une tierce partie, ce qui ne se fait pas sans heurt: "Il se ramasse chez une gardienne et c'est du je m'en foutisme (10) »; "Comment je fais moi, comme parent, pour exiger de ma gardienne qu'elle exige de mon enfant qu'il fasse ses choses (21) »; "Si ça ne va pas à l'école, ça veut pas dire que ça va aller mieux chez la gardienne (109)».

À certains égards, les parents ne trouvent pas leur compte avec la suspension externe et évaluent qu'elle profite davantage au milieu scolaire qu'à leurs enfants : 
Comportements violents chez l'enfant en Ontario:

problématique de la suspension scolaire externe, perception des parents et alternative possible

"Ça aide à l'interne, les professeurs et la direction, de faire une suspension. Ils ont la paix pendant qu'il n'est pas là. Mais, à part eux qui ont des avantages clairs et marqués, je ne pense pas que pour l'enfant, c'est un avantage (11.1); "Ça rend plus service à l'école qu'à nous (44)»; «En attendant qu'on trouve un moyen, je l'ai sorti de la classe pendant un mois parce que ça n'allait pas. Et c'était au grand soulagement des enseignants et de la direction (51)».

Par ailleurs, l'ensemble des parents mentionnent ne pas avoir été consultés avant que l'école prenne la décision de suspendre leur enfant: "L'école t'envoie un papier qui mentionne la suspension et c'est fini. T'as même pas le temps de faire des plans (24) »; "L'école l'a fait sans même m'en parler (47)»; «Ils m'ont appelé pour dire de venir chercher mon enfant immédiatement puisqu'il était suspendu (74)»; "Mon enfant m'a dit qu'il était suspendu pour trois jours lorsqu'il est arrivé à la maison (85)».

Même en ayant manifesté leur désaccord avec la décision de suspendre leur enfant à l'externe, l'école n'a pas tenu compte de l'opinion des parents : "J'ai donné mon avis et on s'en est foutu comme l'an quarante (11)»; "Ils m'ont averti par téléphone. J'ai dit ce que j'en pensais mais ils n'ont pas voulu changer d'idée (46)»; «La direction a maintenu la suspension d'une journée malgré mon désaccord (52)»; "On m’a pas donné le choix (74)».

Des parents mentionnent également que l'école a utilisé des menaces pour maintenir la suspension externe, malgré le profond désaccord exprimé : «Pour me faire accepter la suspension d'une journée, la direction m'a menacée d'imposer trois jours au prochain comportement inacceptable de mon fils. C'est comme du chantage. Finalement, ils font ce qu'ils veulent et nous font des menaces si on ne l'accepte pas (52)»; «Ils m'ont dit, soit tu acceptes qu'on le suspende, soit on le renvoie! (109)».

Par ailleurs, sur l'ensemble des parents en désaccord, seulement trois ont réussi à transformer la suspension externe en suspension interne: "Mon enfant a eu une menace de suspension externe parce qu'il ne voulait pas coopérer avec ses pairs et les adultes. Mais il ne l'a pas eu parce que je n'étais pas d'accord avec cette option. Il a eu seulement une suspension interne (10)»; "Il se faisait suspendre à l'externe. Ils m'ont demandé ce que j'en pensais. Comme je n'étais pas d'accord, elle a trouvé d'autres moyens. Comme par exemple, retirer mon enfant de la classe et le suspendre à l'interne (51)».

Pour certains parents, du fait que la décision de suspendre à l'externe est imposée par l'école, qu'elle va à l'encontre de leur volonté et qu'il est impossible de la faire changer, cette situation leur occasionne un sentiment d'impuissance et d'incompréhension tout en leur faisant ressentir un manque d'emprise sur la situation: "On peut pas faire grand chose, nous autres, on est juste des numéros (06) »; «On a pas de chances les parents. Ils nous écoutent pas (48)»; "Ils m'ont envoyé une feuille où il était écrit que mon fils était suspendu. Si je ne la signais pas, ils l'aurait suspendu de toute manière (85) »; «La direction d'école, y comprennent pas les parents (96)».

Afin de faire face à la situation, des parents réagissent par des gestes de résistance: "J'ai résisté autant que j'ai pu à ce qu'il voulait me faire faire. Finalement, j'ai accepté de donner du Ritalin ${ }^{\circledR}$ à mon fils pour qu'il soit réintégré en classe. Mais, je veux pas lui en donner trop. Je veux pas le rendre zombie. C'est moi qui contrôle le 
Comportements violents chez l'enfant en Ontario:

problématique de la suspension scolaire externe, perception des parents et alternative possible

dosage à l'école. Il n'ont rien en surplus (48)»; «Deux semaines, c'est trop long. [...].. Je leur ai dit que jamais plus je me plierai à leur demande. Jamais! (85)».

À ces difficultés, des parents ajoutent avoir éprouvé des problèmes de communication de toute nature avec le milieu scolaire: "J'ai souvent appelé à l'école pour comprendre ce qui s'était passé en classe. Je restais à l'écoute. On ne peut pas en dire autant d'eux (48)»; "À l'école, quand j'ai questionné l'enseignante, je n'ai pas eu de réponse. [...] Quand j'essayais d'appeler le conseil scolaire, il ne retournait pas mes appels. J'ai été obligé d'écrire une lettre (84)»; "J'aurais aimé être appelé par le prof ou le directeur, qu'ils nous donnent un coup de fil quand quelquechose ne va pas. J'aurais aimé savoir qu'il y avait des problèmes avant que ça devienne assez grave, qu'ils le mettent à la porte (97)».

Pour ajouter à ces difficultés, selon certains parents, l'école a parfois fait preuve d'une attitude blâmante tant à leur égard qu'à celui de leur enfant : "Mon fils s'est fait un mauvais nom à l'école. Ça le suit. Il se fait blâmer souvent (80) »; "Au jardin, il se faisait blâmer jusqu'à ce qu'il y ait une explication de ce qui était arrivé. Quand j'essayais de parler à la direction, tout était de ma faute (84)».

Compte tenu de cette situation, les parents soulignent le besoin criant de ressources humaines spécialisées auxquelles ils pourraient se référer en situation de crise avec l'école : "Il devrait y avoir un système où un psychologue intervient tout de suite. Il y a vraiment un manque de personnel à ce niveau-là (85) »; Ils n'ont pas de conseillère. Ils n'ont personne pour lui parler puis l'aider (97)».

En raison du manque de personnel non enseignant spécialisé en milieu scolaire, les parents soulignent également que la réinsertion de leur enfant en salle de classe n'est pas toujours bien vécue au retour de la suspension: "Le retour à l'école a été très lent. Ma fille allait à l'école trois heures et demi par jour seulement, pendant trois semaines (09) »; «Depuis le retour à l'école, c'est comme... tout est à recommencer (91)».

Malgré leur vive opposition, certains parents se résignent à accepter la suspension externe parce que l'école leur fait part qu'il s'agit d'une loi qui doit être appliquée de manière uniforme et sans distinction pour l'ensemble des élèves : « Même si c'était un événement isolé, elle devait quant même appliquer la politique (01)»; On donne une suspension. On dit que c'est la loi, qu'il faut le faire (12); "L'école m'a dit qu'il était suspendu parce que c'était les règlements de l'école et du conseil. C'était obligatoire et ils ne pouvaient pas faire autrement (83) »; "Ils devaient appliquer la suspension pour respecter la loi de l'Ontario (112)».

Toutefois, aucun des parents en désaccord ne soutient que cette mesure disciplinaire contribue à améliorer les comportements violents de leur enfant. Au contraire, ils suggèrent même qu'elle suscite l'effet inverse: "Je ne pense pas que le système qu'ils ont, de punir constamment les enfants et de les rejeter, non, ça ne donne rien, tant qu'à moi, ça empire la situation (11)»; "Ça fait juste escalader (51)»; "Tout ce que ça cause, c'est que le problème progresse plus vite et devient plus gros (48)»; «Ça peut juste être pire (112)».

Bref, pour toutes ces raisons, les parents ne croient pas à l'efficacité de la suspension externe telle qu'elle appliquée actuellement puisque cette mesure ne permet pas de s'attaquer à la source du problème: "Ma fille a un problème de par- 
Comportements violents chez l'enfant en Ontario :

problématique de la suspension scolaire externe, perception des parents et alternative possible

lage. [...]. La suspension externe est frustrante parce qu'elle représente l'incompréhension de ses problèmes réels (09) »; "Bien, tout ce qu'ils font, c'est suspendre, suspendre, suspendre, parce qu'ils trouvent pas d'autres solutions. Ils n'essaient pas de résoudre le problème (112)».

\section{Les parents plus ou moins en accord}

Parmi les parents plus ou moins en accord avec la suspension externe, certains croient qu'elle serait peut-être appropriée, mais pas à un si jeune âge. Trois de ces parents ont un enfant de 6 ans et moins: "On ne devrait pas utiliser ça avant la première ou la deuxième année et seulement dans les cas sérieux (20) »; "Je trouve qu'à 9 ans, c'est trop jeune pour suspendre (28) »; "À son âge, dans le moment, ça ne lui aide pas du tout (98)»; "Non, pas du tout. Pas à 4 ans. . . Peut-être plus tard, mais pas à 4 ans (108)».

Par contre, d'autres parents semblent penser, au contraire, que plus la suspension externe survient jeune, plus elle a des chances de fonctionner avec l'enfant: "Je pense que plus jeune, ça peut avoir un effet parce qu'ils ont peur de ça (37) »; À son âge, je crois que ça marche parce qu'il n'a pas refait ce qu'il avait fait. Plus tard, ils sont plus contents qu'autre chose (82)».

Selon quelques parents, cette mesure pourrait convenir davantage à certains enfants qu'à d'autres, dépendamment de leur tempérament et de leur personnalité : "Peut-être pour certains enfants plus sensibles, une suspension devient comme un désastre dans son monde à lui. Mais pour mon enfant, ce n'était aucunement un désastre (02) »; "Pour être efficace, ça dépend du caractère de l'enfant (05) »; "Ça peut avoir un effet car il adore aller à l'école. En ce moment, c'est un atout qu'on a réussi à garder. Je dois dire qu'à ce niveau-là, c'est valable (20)».

Quelques parents se sont même laissés convaincre par la direction d'école que cette mesure n'était pas mauvaise en soi pour leur enfant: "Le soir, j'ai parlé à la directrice qui m'a dit de ne pas m'en faire avec ça (05) »; "La directrice m'a rassuré que ça fait partie du développement de l'enfant et qu'il n'y a rien de problématique làdedans (108)».

Bref, bien qu'ils soient plus ou moins en accord avec la suspension externe, des parents évaluent qu'il vaut mieux ne pas s'opposer aux décisions déjà prises par l'école, dans le meilleur intérêt de leur enfant: "Au téléphone, la direction a dit que ça ne pouvait plus continuer comme ça et que la suspension s'en venait. J'ai dit que j'étais d'accord. J'avais pas tellement le choix (28)»; "Ils m'ont demandé ce que j'en pensais, mais je ne voulais pas être contre eux car mon enfant a un problème et c'est comme ça qu'ils règlent ça (98)».

\section{Les parents en accord}

Parmi les parents qui se disent en accord avec la suspension externe, certains pensent qu'elle peut contribuer à responsabiliser leur enfant face aux conséquences de leurs gestes: "Il y a des coups de pied pour lesquels l'enfant doit subir la responsabilité (07)»; "Il doit subir très jeune les conséquences de ses mauvais comportements. Ça l'aidera à devenir un meilleur adulte (23) »; «Dans le fond, la suspension, c'est l'en- 
Comportements violents chez l'enfant en Ontario :

problématique de la suspension scolaire externe, perception des parents et alternative possible

fant qui doit la payer. C'est là où la suspension, oui, je suis d'accord avec ça. Oui, je peux comprendre que vous voulez donner une leçon, mais ce ne doit pas être le parent qui la paie. Ce doit être l'enfant (99)».

D'autres parents pensent que la suspension scolaire peut s'avérer l'élément déclencheur leur permettant d'amorcer un processus de résolutions de la situationproblème: "Ça m'a indiqué qu'il était temps que je me réveille, que ça me pousse à faire quelque chose (12.1)»; "Il y a plusieurs solutions à un problème, puis la changer d'école, c'était juste une des solutions, puis c'était une bonne solution (114)».

Bref, bien que certains parents se disent d'accord avec l'application de la suspension externe, ils expriment qu'ils souhaiteraient avoir une meilleure connaissance de ses effets à long terme: "J'aimerais ça savoir si ça va l'aider tant que ça (14)»; "Je ne sais pas si ça va faire de quoi. J'ai aucune idée, comme j'aimerais ça faire des rencontres, en parler (99)».

\section{Les parents n'ayant pas d'opinions précises}

Tous les parents n'ayant pas d'opinions précises au sujet de la suspension externe expriment que c'est en raison qu'ils ne l'ont pas encore expérimentée : "Je n'ai jamais connu la suspension externe mais seulement une possibilité parce qu'il a un comportement pas mal agressif. Je ne peux pas vraiment dire si ça aiderait ou pas (86) »; «Aucune idée. J'ai pas de commentaires parce que j’ai jamais été obligée de sévir à propos de ça (107)».

Bref, bien qu'ils n'aient pas d'opinions précises au sujet de l'application de cette mesure, les parents considèrent primordial d'être consultés avant qu'elle ne soit mise en application: "Je comprends qu'ils suivent certaines étapes. Je donnerais mon opinion quand même. Je suivrais les indications de la suspension, mais j'essaierais de parler avec l'enseignante et le directeur, en espérant que ça n'arrive plus (30) »; "Même s'ils ont des règles à suivre, l'école devrait toujours me demander mon opinion avant de passer à l'action (32)».

\section{Une alternative : la suspension interne}

Afin de permettre à leur enfant de ne pas entraver leur processus de socialisation et de scolarisation, la majorité des parents de l'échantillon semblent préférer de beaucoup la suspension interne: "Moi je me dis, trois jours de suspension interne, ce serait plus efficace parce qu'il irait à l'école. Puis, il se ferait pas enlever le privilège de voir ses amis (05) »; "Ça serait mieux une suspension interne où ils lui expliqueraient la situation (50); "Il devrait y avoir des suspensions internes à l'école, à un moment où l'enfant peut réfléchir, comme à la récréation ou sur l'heure du dîner (74)»; "La solution, ce serait la suspension scolaire interne. Dans ce cas-ci, il y a beaucoup de choses qui pourraient être essayées, mais en terme de suspension, cela serait interne. Parce qu'il a l'air de bien fonctionner, il est tout seul... au bureau (90)"; "Je crois aussi que les suspensions devraient se passer à l'école, à l'interne (96)». 
Comportements violents chez l'enfant en Ontario:

problématique de la suspension scolaire externe, perception des parents et alternative possible

Toutefois, afin de se révéler efficace, cette mesure disciplinaire ne devrait pas être appliquée seule mais accompagnée d'activités suscitant des prises de conscience : «Moi, je pense qu'un enfant quand il fait quelque chose de mal, il faut le faire parler pour voir pourquoi il a fait ça, puis trouver une solution avec lui (11)»; “Ça dépend de la cause de la suspension. Si l'enfant a besoin de socialiser, la solution n'est pas de l'isoler mais de lui parler (96)».

Selon les parents, la suspension interne devrait également inclure des activités de rattrapage scolaire: "Je suis d'accord avec la suspension interne mais pas dans le bureau du directeur-adjoint parce qu'il perd son temps. Quand il n'est pas en classe, il manque tous les apprentissages. Pourquoi mon fils n'est-il pas installé dans un petit groupe de récupération quand ça va mal? (48)»; "Tu sais, comme lui faire refaire les travaux qu'il manque dans la classe, ou quelque chose comme ça (102)».

De plus, les parents estiment que l'école devrait tenter d'identifier, de manière individuelle, le moyen le plus efficace pour contrer les comportements violents de leur enfant tout en adaptant les conséquences à l'acte selon son tempérament: "L'école devrait plutôt enlever quelque chose que l'enfant aime ou supprimer des privilèges (58) »; "Il faudrait avoir un système après l'école pendant une heure pour qu'ils se rattrapent, en se sentant responsables de leurs actions (85) »; "Je dirais d'enlever des récréations. Mon fils est un petit garçon qui aime aller dehors. Ce serait bon de lui faire perdre ce privilège pour qu'il comprenne (92)».

Les parents suggèrent également que l'école maintienne l'utilisation de techniques qui semblent bien fonctionner en milieu scolaire avec leur enfant: "Il a eu une feuille de comportement et il a passé la récréation à trouver par lui-même pourquoi ça n'allait pas, d'après la méthode Hibou... (12.1)»; "Je trouve que c'est le 1-2-3 magique qui fonctionne le mieux avec lui (13)»; "Le conseil de coopération, c'est magique. Ça fait diminuer ses comportements (90)".

De plus, les parents estiment que l'école aurait avantage à transposer certaines mesures disciplinaires qu'ils utilisent à la maison et qui fonctionnent bien avec leur enfant: "J'ai toujours dit qu'à sa première année, c'était plate pour mon fils. Pas le droit de bouger et de parler. Il faut écouter et se concentrer. Ça marche pas avec lui. Moi, je peux le faire se concentrer sur n'importe quoi parce que je joue avec lui, mais pas l'enseignante (48)»; "Ce que je trouverais important, c'est que les interventions soient concertées. Qu'on puisse le faire à l'école, ce qu'on fait à la maison. Ça je pense que quand c'est travaillé d'une façon comme ça, on a plus de chances que ça marche (14.1)»; "J'utilise l'avertissement, j'annonce une conséquence puis j'attends comme une minute, deux minutes. Si ça marche pas, j'agis... [...] C'est ça qui marche le mieux (108)».

$\mathrm{Au}$ lieu de recourir systématiquement à la suspension, qu'elle soit interne ou externe, les parents proposent d'être partie prenante dans les décisions touchant la vie scolaire de leur enfant : "Qu'ils essayent de fonctionner un peu plus en impliquant les parents (02) »; "Ce qu'il faut faire, c'est de trouver une manière de fonctionner avec l'enfant, en collaboration avec les parents, pour savoir exactement quoi faire quand ça ne va pas à l'école (48)"; "S'il y avait plus de comités parents-direction. [...]. Quelque chose comme un vrai partenariat. En tant que parent, j'irais chercher la voix des autres parents qui sont peut-être pas capables de parler comme moi (99)». 
Comportements violents chez l'enfant en Ontario:

problématique de la suspension scolaire externe, perception des parents et alternative possible

L'application de mesures disciplinaires personnalisées et les prises de décisions communes concernant la situation-problème de l'enfant commandent toutefois davantage d'ouverture et de souplesse que ne le permettent les cadres actuels de la Loi sur la Sécurité dans les écoles (MÉO, 2000a).

\section{Conclusion}

Selon les résultats de la présente recherche, dans sa forme actuelle, la suspension scolaire externe ne semble pas rallier $65 \%$ des parents de la présente recherche, à l'idée qu'elle soit une mesure disciplinaire appropriée pour contrer les comportements violents de leur enfant. Selon eux, son application comporte davantage d'effets négatifs que positifs, en se révélant dommageable au plan de la scolarisation et de la socialisation de leur enfant. Ces résultats se situent dans le prolongement d'études qui concluent à des appréhensions similaires chez les parents (Blyth and Milner, 1993; Hayden, 1994; Royer et al., 1993). Finalement, à l'instar des conclusions de l'étude de Dupper (1994), il s'avère que la suspension externe contribue à générer un contexte conflictuel entre l'école et les parents lorsque ces dernier s'y opposent. Pour toutes ces raisons, les parents semblent privilégier la suspension interne (Farner, 1996; Guindon, 1992; Stage, 1997; Sullivan, 1989). Toutefois, afin d'être formative, ils souhaiteraient que cette mesure disciplinaire ne soit pas appliquée seule mais accom-

La suspension scolaire devrait contribuer à faire développer de nouvelles compétences chez l'élève car le fait de le suspendre en réponse à ses comportements violents envers les pairs à l'école devrait lui permettre de vivre différemment son processus de scolarisation et de socialisation, et non d'en interrompre le cours. pagnée d'activités de rattrapage scolaire et de prises de conscience (Vanderslice, 1999).

Face à cette situation, il y a tout lieu pour l'école d'évaluer dans quelle mesure la suspension externe représente la meilleure solution pour permettre à l'élève de contrer ses comportements violents envers ses pairs. Si cette mesure disciplinaire est privilégiée, elle se doit d'être accompagnée d'un dispositif permettant aux parents de jouer adéquatement leur rôle. En ce sens, le milieu scolaire ne doit jamais perdre de vue que toutes conséquences à l'acte faisant l'objet d'un transfert dans le milieu familial n'a d'impact éducatif que lorsque l'on sauvegarde la valeur de formation qui lui est sous-jacente (Van Neste, 1994).

Par ailleurs, si la suspension interne est privilégiée, il ne faut pas oublier que les conséquences ne sont pas moins graves de maintenir l'enfant violent en milieu scolaire si aucun encadrement ne lui est offert (Hartman et Stage, 2000), puisque celuici risque d'être retardé au plan académique (Feucht, 1998), en plus de faire l'objet de harcèlement et de rejet de la part de ses pairs (Blyth et Milner, 1993). C'est dans cet esprit que les années 1990 ont vu apparaître des programmes de prévention des conduites violentes, axées sur des stratégies de résolution pacifiques de conflits (Garibaldi et al., 1996) et des programmes de développement des compétences sociales (Day et al., 1995). L'argument majeur justifiant le foisonnement de ces nouveaux programmes se fonde sur le fait que les élèves ayant de tels comportements envers les pairs ne possèdent vraisemblablement pas les compétences sociales (Masten et Coatsworth, 1998) leur permettant d'évoluer pacifiquement en milieu scolaire. 
Comportements violents chez l'enfant en Ontario :

problématique de la suspension scolaire externe, perception des parents et alternative possible

Dans un esprit de formation, la suspension scolaire, qu'elle soit externe ou interne, devrait contribuer à faire développer de nouvelles compétences chez l'élève car le fait de le suspendre en réponse à ses comportements violents envers les pairs à l'école devrait lui permettre de vivre différemment son processus de scolarisation et de socialisation, et non d'en interrompre le cours.

\section{Références bibliographiques}

American Psychiatric Association. (1996). The Diagnostic and Statistical Manual of Mental Disorders, $4^{\text {th }}$ Edition. MINI DSM-IV, Critères diagnostiques (Washington, DC, 1994), Traduction française par J.-D. Guelfi et al., Paris : Masson.

Berkowitz, L. (1993). Agression: Its Causes, Consequences, and Control. New York: McGraw-Hill, 485 p.

Bishop, S.J. et Rothbaum, F. (1992). Parents' Acceptance of Control Needs and Preschoolers' Social Behaviour: A Longitudinal Study. Canadian Journal of Behaviour Science, 24(2), 171-185.

Blyth, E. et Milner, J. (1993). Exclusion from School : A First Step in Exclusion from Society? Children \& Society, 7(3), 255-268.

Bouchard, C. et Tessier, R. (1996). Conduites à caractère violent à l'endroit des enfants, Conduites à caractère violent dans la résolution de conflits entre proches, dans Lavallée, C., Clarkson, M. et Chénard, L. (Éds.) Montréal: Santé Québec, 21-76.

Carrington, P.J. (1999). Trends in Youth Crime in Canada, 1977-1996. Canadian Journal of Criminology, 41(1), 1-32.

Christenson, S.L., Hirsch, J.A. et HurIey, C.M. (1997). Families with Aggressive Children and Adolescents, sous la direction de A. Goldstein et J.-C. Conoley, School Violence Intervention : A Practical Handbook. New York : Guilford Press, 325-365.

Conseil canadien de développement social. (2001). Le progrès des enfants au Canada. Ottawa: Conseil canadien de développement social.

Conseil national du bien-être social. (2002). Profil de pauvreté 1999. Seuils de faible revenu après les impôts de Statistique Canada, 117, Ottawa: Ministère des travaux publics et Services gouvernementaux.

Day, D.M., Golench, C.A., MacDougall, J. et Beals-Gonzales, C.A. (1995). La prévention de la violence à l'école au Canada: Résultats d'une étude nationale des politiques et programmes. Ottawa: Solliciteur général du Canada. 
Comportements violents chez l'enfant en Ontario :

problématique de la suspension scolaire externe, perception des parents et alternative possible

Duhamel-Maples, M. (1996). La violence en milieu scolaire: Un défi pour les intervenants et intervenantes. Reflets, Revue ontaroise d'intervention sociale et communautaire, 2, 40-57.

Dupper, D.R. (1994). Reducing Out-of-School Suspensions : A Survey of Attitudes and Barriers, Social Work in Education, 16(2), 115-123.

Dupper, D.R. et Bosch, L.A. (1996). Reasons for School Suspensions. Journal for a Just \& Caring Education, 2(2), 140-150.

Farner, C.D. (1996). Proactive Alternatives to School Suspension. Journal of Emotional \& Behavioral Problems, 5(1), 47-51.

Fasko, D., Grubb, D.J. et Osborne, J.S. (1997). Suspensions of Students with and without Disabilities: A Comparative Study, Research in the Schools, 4(1), 45-50.

Feucht, J.E. (1998, March). Reading, Writing, Arithmetic, and Suspensions. NASSP Bulletin, 82, 14-15.

Fortin, L., Toupin, J., Pauzé, R., Déry, M. et Mercier, H. (1996). Variables associées à la compétence scolaire des adolescents en troubles de comportement. Scientia paedagogica experimentalis, 23(2), 245-268.

Gagnon, C. et Vitaro, F. (2000). La prévention du trouble des conduites, avec centration sur les comportements violents, in Vitaro, F. et Gagnon, C. Prévention des problèmes d'adaptation chez les enfants et les adolescents. Tome II : Les problèmes externalisés. Ste-Foy: Presses de l'Université du Québec, 231-290.

Garibaldi, A., Blanchard, L. et Brooks, S. (1996). Conflict Resolution Training, Teacher Effectiveness, and Student Suspension: The impact of a Health and Safety Initiative in the New Orleans Public Schools. Journal of Negro Education, 65(4), 408-413.

Guindon, J. (1992). Developing an In-School Suspension Program in an Elementary School as an Alternative to Home-Bound Suspension. Practicum Report, CT : Nova University.

Hartman, R. et Stage, S.A. (2000). The relationship Between Social Information Processing and In-school Suspension for Students with Behavioral Disorders, Behavioral Disorders, 25(3), 183-195.

Hayden, C. (1994). Primary Age Children Excluded from School : A multi Agency Focus for Concern. Children \& Society, 8(3), 257-273.

Imich, A.J. (1994). Exclusions from School : Current Trends and Issues. Educational Research, 36(1), 3-11.

Jeffrey, D. et Simard, C. (2000). Enseigner et punir. Québec: Presses de l'Université Laval, p. 38-42.

Kauffman, J.M. (1997). Characteristics of Emotional and Behavioral Disorders of Children and Youth. (6 $6^{\text {th }}$ Ed.). Upper Saddle River, N.J. : Merrill, Prentice-Hall. 
Comportements violents chez l'enfant en Ontario :

problématique de la suspension scolaire externe, perception des parents et alternative possible

Kratzer, L. et Hodgins, S. (1997). Adult outcomes of child conduct problems : A cohort study. Journal of Abnormal Child Psychology, 25, 65-81.

Lauwrence, B. et Hayden, C. (1995). Primary School Children Excluded from School: Numbers, Characteristics, Reasons and Circumstances. Paper presented at the European Conference on Educational Research. Bath, England, U.K.

Leblanc, M. (1999). La délinquance des adolescents, dans F. Dumont, S. Langlois et Y. Martin, Traité des problèmes sociaux. Sainte-Foy: Presses de l'Université Laval, 279-299.

L'Écuyer, R. (1987). L'analyse de contenu: Notion et étapes, La recherche qualitative: Résurgence et convergences, Jean-Pierre Deslauriers (Éd.), Chicoutimi : Université du Québec à Chicoutimi, 65-88.

Lemonick, M.D. (2002). Germany's Columbine. Time, Canadian Edition, May, 6, p. 26-27.

Loeber, R. et Farrington, D.P. (2000). Young children who commit crime : Epidemiology, developmental origins, risk factors, early interventions and policy implications, Development and Psychopathologie, 12, 737-762.

Masten, A.S. et Coatsworth, J.D. (1998). The development of competence in favorable and unfavorable environments. Lessons from research on successful children. American Psychologist, 53(2), 205-220.

Mellard, D. et Seybert, L. (1996). Voices about School Suspension, Expulsion, and Learning. Final Report, Topeka : Kansas State Board of Education.

Ministère de l'Éducation de l'Ontario. (2001a). Les programmes de discipline rigide. Document d'information, Toronto : Gouvernement de l'Ontario.

Ministère de l'Éducation de l'Ontario. (2001b). Éducation de l'enfance en difficulté: Guide pour les éducatrices et éducateurs. Partie A : Lois et politiques. Toronto : Gouvernement de l'Ontario.

Ministère de l'Éducation de l'Ontario. (2000a). L'Ontario fixe des normes pour accroître la sécurité dans les écoles. Document d'information, Toronto : Gouvernement de l'Ontario.

Ministère de l'Éducation de l'Ontario. (2000b). Écoles de l'Ontario: Code de conduite provincial. Document d'information, Toronto : Gouvernement de l'Ontario.

Ministère de l'Éducation du Québec. (1992). Interprétation des définitions des élèves handicapés ou en difficulté d'adaptation ou d'apprentissage. Québec : Gouvernement du Québec.

Ministère de l'Éducation et de la Formation de l'Ontario. (1994). Pour des écoles sans violence: Une politique, Toronto : Gouvernement de l'Ontario.

Ministère de la Justice du canada. (1998). La Stratégie fédérale en matière de justice pour les jeunes. Document d'information. Ottawa: Gouvernement du Canada. 
Comportements violents chez l'enfant en Ontario :

problématique de la suspension scolaire externe, perception des parents et alternative possible

Ministère de la Justice du canada. (2002). La Loi sur le système de justice pénale pour les adolescents: Une nouvelle loi, une nouvelle approche. Document d'information. Ottawa: Gouvernement du Canada.

Nagin, D.S. et Tremblay, R.E. (2001). Parental and Early Childhood Predictors of Persistent Physical Aggression in Boys From Kindergarten to High School. Arch Gen Psychiatry, 58, 389-394.

Paquin, M. et Drolet, M. (2000). Les conceptions contemporaines de l'enfance à risque : Commentaires critiques. L'enfance et la jeunesse à risque. Ottawa : Conseil des ministres de l'éducation du Canada, 8, 35.

Patterson, G.R. (1982). A Social Learning Approach to Family Intervention. Chapter III : Coercive Family Process. Eugene, OR: Castalia.

Rich, J. M. (1992). Predicting and controlling school violence. Contemporary Education, 64, 35-39.

Stage, S.A. (1997). A Preliminary Investigation of the Relationship Between In-School Suspension and the Disruptive Classroom Behavior of Students with Behavioral Disorders. Behavioral Disorders, 23(1), 57-76.

Strauss, M.A. et Gelles, R.J. (1990). Physical Violence in American Families : Risk Factors and Adaptations to Violence in 8,145 Families. N.B. : Transaction Publishers.

Sullivan, J.S. (1989). Elements of a Successful In-School Suspension Program. NASSP Bulletin, 73, 32-38.

Uchitelle, S., Bartz, D. et Hillman, L. (1989). Strategies for Reducing Suspensions. Urban Education, 24(2), 163-176.

Tremblay, R.E. (1995). Les enfants violents à l'école primaire : Qui sont-ils et que deviennent-ils? Dimensions, 36-37.

Vanderslice, R. (1999). Developing Effective In-School-Suspension Programs. Paper presented at the Annual Meeting of Women in Educational Leadership. Bicton, Western Australia.

Van Neste, M. (1994). La réussite éducative et la suspension de l'élève : Un antagonisme irréconciliable? Vie Pédagogique, 80, 25-28.

Viau, Y. (2001). Le Canada renouvelle son système de justice pour les jeunes. Hull : Actes du Symposium international sur la jeunesse, 40-43.

Vitaro, F. et Gagnon, C. (2000). Prévention des problèmes d'adaptation chez les enfants et les adolescents. Tome II : Les problèmes externalisés. Ste-Foy: Presses de l'Université du Québec. 